Pacific Journal of Mathematics

ACCEPTABLE POINTS IN GAMES OF PERFECT 


\title{
ACCEPTABLE POINTS IN GAMES OF PERFECT INFORMATION
}

\author{
ROBERT J. AUMANN
}

Summary. This is the second of a series of papers on the theory of acceptable points in $n$-person games. The first was [1]; in it the notion of acceptable points was defined for cooperative games, and a fundamental theorem was proved relating the acceptable expected payoffs for a single play of a game to probable average payoffs for "strong equilibrium points" in its supergame. ${ }^{1}$

The chief result of the current paper, Theorem 5.4, is a generalization of von Neumann's classical Theorem on two-person zero-sum games of perfect information (see [11]). Roughly, it states that strong equilibrium points in the supergame of a stable game of perfect information can be achieved in pure supergame strategies. An example shows that not all games possess this property; and in fact, it is conjectured that the property is characteristic of game structures of perfect information.

The theorem stated above holds whether $G$ is interpreted as a cooperative or as a non-cooperative game. To lend meaning to this statement, we will have to extend the theory introduced in [1] to non-cooperative games. We plan to do this in full in a subsequent paper. Here just enough definitions and theorems will be used to enable us to state and prove the chief result for non-cooperative games of perfect information.

The paper is divided into two parts, the first centering around the proof of the chief result for cooperative games, the second dealing with the extension to non-cooperative games. Section 1, the introduction, serves mainly to supply background from [1] and from the literature. In $\S 2$, we show that the naive approach to generalizing von Neumann's theorem on games of perfect information fails; that is, we bring an example of a stable game of perfect information that has no acceptable point in pure strategies. It is then shown intuitively that an appropriate generalization of the von Neumann Theorem should involve the supergame. Sections 3 and 4 are devoted to the proof of preliminary theorems, dealing with supergame pure strategies and supergames of perfect information, respectively. In $\S 5$ we establish the chief result. Section 6, which completes the first part of the paper, is devoted to the example and conjecture mentioned in connection with the chief result.

Received September 4, 1958.

${ }_{1}^{1}$ Readers not familiar with $[\mathbf{1}]$ should read the introduction (section 1 ) before continuing with this summary. 
The second part begins with $\S 7$, a summary of the additional notation needed for the non-cooperative case. In $\S 8$ the concept of acceptability is defined for non-cooperative games. In $\S 9$, we show that in a game $G$ of perfect information, it makes no difference, insofar as the theory of acceptable points is concerned, whether $G$ is to be considered as a non-cooperative or as a cooperative game. More precisely, it is shown that the set of acceptable payoffs in the non-cooperative sense, coincides with the set of acceptable payoffs in the cooperative sense. This is a consequence of the lemma, interesting in its own right, that in a game of perfect information, the set of payoff vectors to correlated strategy vectors coincides with the set of payoff vectors to mixed strategy vectors. Again, this lemma seems to be characteristic of game structures of perfect information. In $\S 10$, we define supergame strategies for non-cooperative games and prove some preliminary results. Section 11 is devoted to the statement and proof of the chief result for noncooperative games of perfect information.

As in [1], the games under consideration contain no chance moves.

We will make unrestricted use of the notations, ideas, definitions, theorems and proofs of [1]. We will not in general repeat explanations and proofs that are similar to those given there. Especially heavy use will be made of $\S 6$ of [1].

1. Introduction and background. Up to the present, the starting point for all work on games of perfect information has been the theorem of von Neumann that every two-person zero-sum game of perfect information with finitely many moves has a solution in pure strategies. Subsequent work has dealt with extensions to $n$-person games and the concomitant generalizations of the solution notion, with various converses to the von Neumann theorem, with extensions to games containing infinitely many moves (i. e., positions), and with various combinations of these. We mention also the notion of stochastic games of perfect information with infinitely many moves.

In the first of these areas, Kuhn [9] showed that the von Neumann theorem could be extended to $n$-person games if the "equilibrium point" notion of Nash [12] was substituted for the classical solution notion. Dalkey [4] proved a converse of this theorem, which reduces to a converse of the von Neumann theorem in the two-person, zero-sum case. Gale and Stewart [6] were the first to treat games of perfect information with infinitely many moves; they showed that certain such (twoperson zero-sum) games possess no pure strategy solutions, and derived sufficient conditions for the existence of a pure strategy solution. Wolfe [14] extended their results. By adopting a definition of payoff that is somewhat more restricted than that of Gale and Stewart, Berge [2] was able to extend von Neumann's theorem to some games with infinitely 
many moves. He was also able to show $[2,3]$ that under very general conditions on the structure of the game, Kuhn's theorem on the existence of a pure strategy equilibrium point in a game of perfect information holds true. The work of Shapley [13] and Gillette [7] on Stochastic Games of perfect information will be discussed in detail below.

The current paper deals with an extension of the von Neumann theorem to $n$-person games. The solution notion that we use is that of "acceptable" points, introduced in [1]. The notion of acceptability is a generalization of the "core" introduced by Gillies [8] for the cooperative game with side payments. More precisely, an $n$-tuple $x$ of strategies is called acceptable if the players of any given coalition can be prevented by the players not in that coalition from each obtaining a higher payoff than when $x$ is played (Definition 4.1 of [1]). Intuitively, it would seem that in a long sequence of plays of a game, a "steady state" would have to represent an acceptable point, because the players would certainly tend to move away from any point that is not acceptable.

In order to obtain a precise statement and proof of this intuitive idea, we introduced (in $\S 6$ of [1]) the formal notion of the "supergame" of a given game $G$. The supergame of $G$ is a game each play of which consists of an infinite sequence of plays of $G$. The payoff to a superplay (i.e., a play of the supergame) is given by the average (i.e., first césaro limit, if it exists) of the payoffs to the individual plays of $G$ that constitute the superplay. Many of the notions that apply to ordinary games can also be applied to supergames. In particular, it is possible to define the notion of strategy in the supergame, and also the notion of a strategy equilibrium point in the sense of Nash. A much stronger form of the Nash equilibrium notion may be defined as follows: An $n$-tuple $x$ of strategies is called a "strong equilibrium point" if for no coalition $B$ can all the members of $B$ increase their payoff by adopting strategies different from those at $x$ while the remaining players (those in $N-B$ ) play as they did at $x$. The notion of strong equilibrium applied to the supergame provides a formalization of the "steady state" idea $(\S 7$ of $[1])$.

The basic result of $[1](\S 10)$ may be stated as follows: The payoffs for the acceptable points in a game $G$ are the same as the payoffs for the strong equilibrium points in its supergame. Since the notion of acceptability depends only on the payoff, this means that the acceptable points in $G$ correspond precisely to the steady state points in the supergame of $G$. For two-person zero-sum games, a point is acceptable if and only if its payoff is the game value, whereas it is a strong equilibrium point if and only if it is a'solution ( $\$ 5$ of $[\mathbf{1}]$ ).

The object of this paper is to apply the theory of acceptable points to games of perfect information, with a view to obtaining an appropriate 
$n$-person generalization of the von Neumann theorem. In other words, we want to accomplish for acceptable points in games of perfect information what Kuhn did in [9] for equilibrium points in games of perfect information. The first conjecture in this direction might be that every game of perfect information has an acceptable point in pure strategies. This is unreasonable, because according to an example given in [1] (\$11), not every game of perfect information need have an acceptable point at all, let alone one in pure strategies. However, it turns out that not even all stable games (games that do have acceptable points) of perfect information have pure strategy acceptable points. The reasons for this are discussed in $\S 2$, and it is also shown there that a more appropriate place to look for a generalization of the von Neumann theorem is in the supergame. We would like to show that if $G$ is a game of perfect information, then each player can restrict himself to pure strategies in each play of an infinite sequence of plays of $G$. In fact, we prove (Theorem 5.4) that every acceptable point (and hence every strong equilibrium point) in a game of perfect information can be "achieved" in pure supergame strategies, in the sense that there is a pure strategy strong equilibrium point with the same payoff. In particular, if the supergame of a game of perfect information has a strong equilibrium point at all, then it already has one in pure strategies.

Formally, the supergame defined in [1] bears some resemblance to the stochastic games treated by Gillette in [7]. The two concepts are similar in that both involve games consisting of an infinite sequence of plays of finite games, and the payoffs in both cases are given by a form of the average of the payoffs to the individual plays. The main differences are that Gillette considers a set of $M$ games, any one of which may be the game played at a given stage, whereas we are concerned with repeated plays of one game only; and that Gillette considers twoperson zero-sum games, while we deal with $n$-person games. The "intersection" of the two theories is an infinite sequence of plays of the same two-person zero-sum game of perfect information, a trivial situation once von Neumann's theorem is known (obviously both players play their optimal pure strategies on each play). The two theories provide totally "disjoint" generalizations of the von Neumann theorem.

All of Gillette's positive results involve "stationary" strategies, i.e., supergame strategies that are obtained by repeating the same strategy on each play of the infinite sequence of plays that constitutes a superplay. In a somewhat similar situation, Everett [5] gives a formal definition of some strategies that are not stationary, and obtains positive results with them; but the strategies he defines are still "almost" stationary in the sense that the choice of a player at a given game of the supergame can depend only on which game he is at, not on the 
choices of the other players on previous occasions.

It is of interest to ask whether these restricted notions of strategy are sufficient for our theory. The answer is no. The existence of a strong equilibrium point in stationary pure strategies would imply the existence of an acceptable point in pure strategies; and the example in $\S 2$ shows that even in stable games of perfect information such an acceptable point in pure strategies need not always exist. The same example shows that there is no strong equilibrium point in "almost" stationary pure strategies.

Finally, we mention that in the supergames of games of perfect information (even unstable ones), there is always a Nash equilibrium point (as opposed to a strong equilibrium point) in stationary pure strategies. This is a consequence of Kuhn's theorem.

2. Failure of the naive approach. We saw in $[1](\S 5)$ that the concept of acceptability constitutes a generalization of the concept of solution in two-person, zero-sum games. As a generalization of Von Neumann's Theorem on two-person zero-sum games of perfect information, we might hope that every game of perfect information that has any acceptable points also has acceptable points in pure strategies. An example shows that this is false.

The game $G$ is a two-person, non-zero-sum game of perfect information. In the game tree, given in Figure 1, the moves are labelled with the names of the players and the terminals with the payoff vectors.

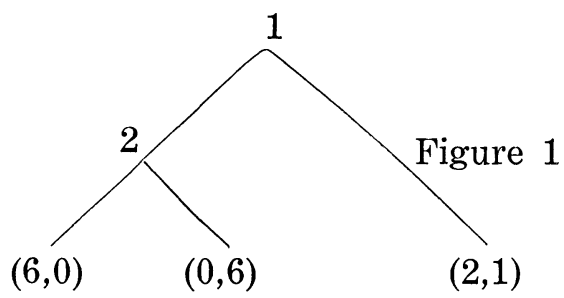

Each player has two strategies, the left and the right strategies. Notation in the following payoff matrix is obvious.

$\begin{array}{lcc} & L^{1} & R^{1} \\ L^{2} & (6,0) & (2,1) \\ R^{2} & (0,6) & (2,1)\end{array}$

Player 1 cannot be prevented from obtaining at least 2 (he can play $R^{1}$ ); player 2 cannot be prevented from obtaining at least 1 (he can play $R^{2}$ ). This shows that $\left(L^{1}, L^{2}\right)$ and $\left(L^{1}, R^{2}\right)$ are not acceptable. The other two pure strategy pairs are not acceptable because the coalition $(1,2)$ cannot be prevented from obtaining $(3,3)$-by playing $\left(L^{1}, 1 / 2 L^{2}+1 / 2 R^{2}\right)$-and $(3,3)$ is strictly larger than the payoff vector at both $\left(R^{1}, L^{2}\right)$ and $\left(R^{1}, R^{2}\right)$. 
Hence $G$ has no acceptable point in pure strategies. (Note that $(3,3)$ is an acceptable payoff vector, so that $G$ does have some acceptable points.)

The intuitive feeling that a game of perfect information should have a "good" point in pure strategies can be traced to the traditional purpose of mixed strategies-namely, to hide one's intensions from one's opponent by the use of a random device. In a game of perfect information, we somehow feel that it is unnessary to hide one's intensions, that it is in the nature of the game that everything may just as well be open and above-board. The conclusion is that mixed strategies are unnecessary in such a game, and that therefore we may just as well confine ourselves to the consideration of pure strategies.

The counter example points up the fallacy in this intuitive argument. It is quite true that the hiding of one's intentions, and the concommitant use of a random device should be unnecessary in a game of perfect information. This does not mean, though, that one can achieve one's desires by means of pure strategies. Indeed, if there were some means of mixing one's strategies other than by the use of a random device, this would be perfectly satisfactory in Example 3. For example, the pair $\left(L^{1}, 1 / 2 L^{2}+1 / 2 R^{2}\right)$ happens to be acceptable. If, instead of tossing a coin before each play of a sequence of plays, 2 were to announce beforehand that he will alternate $L^{2}$ and $R^{2}$, this would in no way affect the actions of 1 . Contrary to the situation in, say, penny matching, the purpose of playing $1 / 2 L^{1}+1 / 2 R^{1}$ here is simply to achieve a payoff not provided in the matrix, not to avoid "discovery" by the opponent.

This discussion shows that though we cannot expect pure-strategy acceptable points in a game $G$ of perfect information, we should be able to expect that the players may, without loss, restrict themselves to pure strategies in each of the plays that constitute a superplay of $G$. This is in fact the case, as we shall see in the sequel.

3. Supergame pure strategies. A supergame pure strategy vector (or $p$-strategy vector) is a $c$-strategy vector in which there are no coalitions and the players choose pure strategies on each play. Here the second condition is the essential one; the first condition is adopted only for convenience. If the first condition were eliminated, the resulting supergame strategy vectors would be essentially equivalent to those obtained under our definition.

The formal definition is as follows:

Definition 3.1. A supergame c-strategy $f^{i}$ is said to be "pure" if

$$
f_{k}^{i}(y) \in P^{i}
$$

for each $k \geq 0$ and $y \in J_{1}^{i} \times \cdots \times J_{k}^{i}$. 
We also say that $f^{i}$ is a supergame $p$-strategy.

The following are lemmas that will be needed later.

LEMma 3.2. If $f$ is a supergame p-strategy vector and $B$ is a (possibly empty) subset of $N$, then for each $k \geq 0$ and $y \in J_{1} \times \cdots \times J_{k}$, we have

$$
f_{k}^{N-B}(y) \mid R^{N-B}=d_{e}^{N-B} .
$$

Furthermore, for each $k \geq 1$, we have

$$
z_{k}(f)=\left(x_{1}(f), \cdots, x_{k}(f)\right) .
$$

Proof. The first statement follows at once from 3.1. The second statement follows by induction from $6.3,6.4$, and 6.5 of [1], and from 3.1 .

Lemma 3.3. Let $f$ be a supergame p-strategy vector, and let $g$ be a supergame c-strategy vector for which

$$
g^{N-B}=f^{N-B} .
$$

Let $v=\left(v_{1}, \cdots, v_{k}, \cdots\right) \in J_{1} \times \cdots \times J_{k} \times \cdots$ occur with positive probability when $g$ is played (see definition 10.22 of [1]). Then for all $k \geq 1$, we have

$$
v_{k} \mid R^{N-B}=d_{e}^{N-B}
$$

and for all $k \geq 0$, we have

$$
f_{k}^{N-B}\left(v_{1}, \cdots, v_{k}\right)=f_{k}^{N-B}\left(\left(v_{1} \mid U_{1}, d_{e}\right), \cdots,\left(v_{k} \mid U_{k}, d_{e}\right)\right) .
$$

Proof. The first statement is an immediate consequence of the previous lemma. As for the second statement, it follows from the first statement that

$$
\begin{aligned}
& f_{k}^{N-B}\left(v_{1}, \cdots, v_{k}\right)=f_{k}^{N-B}\left(\left(v_{1}^{B}, v_{1}^{N-B}\right), \cdots,\left(v_{k}^{B}, v_{k}^{N-B}\right)\right) \\
& \quad=f_{k}^{N-B}\left(\left(v_{1}^{B},\left(v_{1}^{N-B} \mid U_{1}^{N-B}, d_{e}^{N-B}\right)\right), \cdots,\left(v_{k}^{B},\left(v_{k}^{N-B} \mid U_{k}^{N-B}, d_{e}^{N-B}\right)\right)\right) .
\end{aligned}
$$

But by Definition 6.1 of $[1], f_{k}^{N-B}$ is independent of $\left(v_{1}^{B}, \cdots, v_{k}^{B}\right)$. The result follows at once.

For a supergame $c$-strategy vector $f$, define $S_{k}(f)\left(=S_{k}\right)$ by

$$
S_{k}(f)=\frac{1}{k} \sum_{j=1}^{k} H_{j}(f) .
$$

Parallel to the definition of strong equilibrium $c$-point, we may make the following definition: 
DEFINITION 3.5. A strong equilibrium p-point $f$ is a summable supergame p-strategy vector for which there is no $B \subset N$ for which there is a supergame p-strategy vector $g$ satisfying

$$
g^{N-B}=f^{N-B}
$$

and

$$
\limsup _{k \rightarrow \infty} \min _{i \in B}\left(S_{k}^{i}(g)-H^{i}(f)\right)>0 .
$$

The set of all strong equilibrium $p$-points is denoted by $S_{p}$. The Condition 3.7 may also be replaced by the following condition:

$$
\liminf _{k \rightarrow \infty}\left(S_{k}^{B}(g)-H^{B}(f)\right)>0 .
$$

We denote by $\widetilde{S}_{p}$ the set of supergame $p$-strategy vectors that satisfy a condition that differs from 3.5 only in that 3.7 is replaced by 3.8 .

The essential difference between a strong equilibrium $p$-point and a pure strong equilibrium $c$-point is that in the former, $N-B$ need only be prepared to defend against all supergame pure strategy $B$-vectors, whereas in the latter, $N-B$ must be prepared to defend against all supergame correlated strategy $B$-vectors. We will show in 3.11 that the two conditions are nevertheless equivalent. As for 3.7 and 3.8, they are merely translations of 7.2 and 7.3 of [1] to the case of pure strategies, where the consideration of probabilities becomes superfluous.

THeOREm 3.9. If $f$ is a supergame p-strategy vector, then $z_{k}(f)$, $x_{k}(f)$, and $E_{k}(f)$ are "pure" for each $k \geq 0$; that is, they are discrete probability distributions in which one of the events occurs with probability 1 , all others with probability 0 .

Proof. This is a trivial consequence of (6.2), (6.3), (6.4), (6.5) and (6.6) of [1], and of 3.1 .

Theorem 3.9 enables us to replace probability statements involving the random variable $S_{k}(v)$ by statements involving the constants $S_{k}(f)$ only. More precisely, we have

CoRollary 3.10. Let $F\left(x_{1}, x_{2}, \cdots\right)$ be a predicate depending on a sequence of $B$-vectors $x_{1}, x_{2}, \cdots$. Let $A$ be the proposition a function that assigns the number 1 to true propositions and the number 0 to false propositions. Suppose $f$ is a supergame p-strategy vector for the game G. Then

$$
\operatorname{Prob}_{f} F\left(S_{1}^{B}(v), S_{2}^{B}(v), \cdots\right)=A\left(F\left(S_{1}^{B}(f), S_{2}^{B}(f) \cdots\right)\right)
$$

Similar results hold for $z_{k}(f), x_{k}(f)$ and $E_{k}(f)$. 
THEOREM 3.11. Every strong equilibrium p-point is a strong equilibrium c-point. Conversely, every pure strong equilibrium c-point is a strong equilibrium p-point. In symbols

$$
F_{p} \cap S_{c}=S_{p}
$$

where $F_{n}$ is the set of supergame p-strategy vectors.

Proof. We consider first the converse, the easier of the two statements. Let $f$ be a pure strong equilibrium $c$-point. It is sufficient to prove that there is no pure $g$ satisfying 3.6 and 3.7. Suppose there is such a $g$. Then $g$ must satisfy 7.1 of $[1]$, which is identical with 3.6. Furthermore, from 3.7 we deduce the existence of an $\varepsilon>0$ such that for infinitely many $k$, we have

$$
\min _{i \in B}\left(S_{k}^{i}(g)-H^{i}(f)\right)>\varepsilon .
$$

It follows that for infinitely many $k$, we have

$$
S_{k}^{B}(g)>H^{B}(f)+\varepsilon^{B},
$$

where $\varepsilon^{B}$ is a $B$-vector defined by

$$
\varepsilon^{i}=\varepsilon
$$

for all $i \in B$. Hence it follows that for all $k$, we have

$$
S_{r}^{B}(g)>H^{B}(f)+\varepsilon^{B} \text { for some } r \geq k .
$$

Applying 3.10, we obtain

$$
\operatorname{Prob}_{g}\left(S_{r}^{B}(v) \geq H^{B}(f)+\varepsilon^{B} \text { for some } r \geq k\right)=1
$$

for all $k$. Hence it follows that

$$
\lim _{k \rightarrow \infty} \operatorname{prob}_{g}\left(S_{r}^{B}(v) \geq H^{B}(f)+\varepsilon^{B} \text { for some } r \geq k\right)=1>0 .
$$

But this is exactly Condition 7.2 of $[1]$. We have established that $g$ satisfies 7.1 and 7.2 of [1], whence $f$ cannot be a strong equilibrium $c$-point. This contradicts the hypothesis, and we must conclude that $g$ satisfies 3.6 and 3.7. This completes the proof of the converse.

Now assume that $f$ is a strong equilibrium $p$-point, but not a strong equilibrium $c$-point. Then there is a supergame $c$-strategy vector $g$ satisfying 7.1 and 7.2 of [1]. From 7.1 of [1] we obtain

$$
g^{N-B}=f^{N-B} \text {. }
$$

From 7.2 of [1], we obtain that there is a $B$-vector $\varepsilon^{B}>0$ for which

$$
\lim _{k \rightarrow \infty} \operatorname{Prob}_{g}\left(S_{r}^{B}(v) \geq H^{B}(f)+\varepsilon^{B} \text { for some } r \geq k\right)>0 .
$$


Now the expression inside the limit on the left side of (2) is monotone decreasing with $k$; hence (2) implies the existence of a

$$
\delta>0
$$

such that

(4) $\operatorname{Prob}_{g}\left(S_{r}^{B}(v) \geq H^{B}(f)+\varepsilon^{B}\right.$ for some $\left.r \geq k\right)>\delta$, for all $k \geq 1$.

From (4) we obtain

(5) $\operatorname{Prob}_{g}\left(\right.$ For all $k \geq 1, S_{r}^{B}(v) \geq H^{B}(f)+\varepsilon^{B}$ for some $\left.r \geq k\right) \geq \delta$, which is the same as

$$
\operatorname{Prob}_{g}\left(S_{r}^{B}(v) \geq H^{B}(f)+\varepsilon^{B} \text { for infinitely many } r\right) \geq \delta .
$$

That (5) follows from (4) is an immediate consequence of the fact that the measure of the intersection of a monotone decreasing sequence of measurable sets is the limit (or g.l.b.) of the measures of the sets.

From (3) and (6) it follows that there is a sequence

$$
v=\left(v_{1}, \cdots, v_{k}, \cdots\right) \in J_{1} \times \cdots \times J_{k} \times \cdots,
$$

occurring with positive probability when $g$ is played, for which

$$
S_{r}^{B}(v) \geq H^{B}(f)+\varepsilon^{B} \text { for infinitely many } r .
$$

Since $v$ occurs with positive probability we deduce from 6.4 and 6.2 of [1], and from the definitions in $\S 2$ of [1] that for each $k$,

$$
\begin{aligned}
0< & s\left(g_{k-1}\left(v_{1}, \cdots, v_{k-1}\right)\right)\left(v_{k}\right) \leq u\left(c\left(g_{k-1}\left(v_{1}, \cdots, v_{k-1}\right)\right)\right)\left(v_{k} \mid U_{k}\right) \\
& =\sum_{p_{k} \in u^{-1}\left(v_{k} \mid U k\right)} c\left(g_{k-1}\left(v_{1}, \cdots, v_{k-1}\right)\right)\left(p_{k}\right) .
\end{aligned}
$$

It follows that for each $k$, there is a $p_{k}$ satisfying

$$
v_{k} \mid U_{k}=u\left(p_{k}\right)
$$

and

$$
c\left(g_{k-1}\left(v_{1}, \cdots, v_{k-1}\right)\right)\left(p_{k}\right)>0 .
$$

Now as a consequence of 2.7 of [1], Lemmas 3.2 and 3.3, and (1), we have that for each $k \geq 0$,

$$
c^{N-B}\left(g_{k}\left(v_{1}, \cdots, v_{k}\right)\right)=g_{k}^{N-B}\left(v_{1}, \cdots, v_{k}\right) .
$$

From (9) it follows that

$$
c^{N-B}\left(g_{k}\left(v_{1}, \cdots, v_{k}\right)\right)\left(p_{k+1}^{N-B}\right)>0 .
$$

Applying (10), we deduce that 


$$
g_{k}^{N-B}\left(v_{1}, \cdots, v_{k}\right)\left(p_{k+1}^{N-B}\right)>0,
$$

and it then follows from (1) that

$$
f_{k}^{N-B}\left(v_{1}, \cdots, v_{k}\right)\left(p_{k+1}^{N-B}\right)>0 .
$$

Since $f_{k}^{N-B}$ must be a pure strategy $(N-B)$-vector, it follows from (11) that

$$
f_{k}^{N-B}\left(v_{1}, \cdots, v_{k}\right)=p_{k+1}^{N-B} .
$$

We now define a supergame $p$-strategy vector $q$ by

$$
\begin{gathered}
q^{N-B}=f^{N-B} \\
q_{k-1}^{i}=p_{k}^{i}, \quad i \in B, k \geq 1 .
\end{gathered}
$$

Next, we prove that for $k \geq 1$,

$$
z_{k}(q)=\left(\left(v_{1} \mid U_{1}, d_{e}\right), \cdots,\left(v_{k} \mid U_{k}, d_{e}\right)\right) .
$$

That

$$
z_{k}(q) \mid R_{1} \times \cdots \times R_{k}=\left(d_{e}, \cdots, d_{e}\right)
$$

follows at once from (13), (14) and the fact that $f$ is a supergame $p$-strategy vector. The remainder of (15) is proved by induction on $k$. For $k=1$, we have by 6.2 and 6.3 of [1],

$$
\begin{aligned}
z_{1}(q) \mid U_{1} & =u\left(c\left(q_{0}\right)\right) \\
& =u\left(c\left(p_{1}^{B}, f_{0}^{N-B}\right)\right)(\text { by }(13) \text { and }(14)) \\
& =u\left(c\left(p_{1}\right)\right)(\text { by }(12)) \\
& =u\left(p_{1}\right)(\text { by } 2.7 \text { of }[1]) \\
& =v_{1} \mid U_{1} .
\end{aligned}
$$

Now let us assume that we have established

$$
z_{k}(q) \mid U_{1} \times \cdots \times U_{k}=\left(v_{1}\left|U_{1}, \cdots, v_{k}\right| U_{k}\right) .
$$

Then by 6.2 and 6.4 of [1],

$$
z_{k+1}(q) \mid U_{1} \times \cdots U_{k+1}=\sum_{y \in J_{1} \times \cdots \times J_{k}}^{*} z_{k}(q)(y)\left(y \mid U_{1} \times \cdots \times U_{k}, u\left(c\left(q_{k}(y)\right)\right)\right) .
$$

By (16) and (18), all the coefficients $z_{k}(q)(y)$ in this sum vanish, unless

$$
y=\left(\left(v_{1} \mid U_{1}, d_{e}\right), \cdots,\left(v_{k} \mid U_{k}, d_{e}\right)\right) \text {. }
$$

Hence

$$
z_{k+1}(q) \mid U_{1} \times \cdots \times U_{k+1}=\left(v_{1}\left|U_{1}, \cdots, v_{k}\right| U_{k}, u\left(c\left(q_{k}\left(z_{k}(q)\right)\right)\right)\right) .
$$

Now by (14), 


$$
q_{k}^{B}\left(z_{k}(q)\right)=p_{k+1}^{B}
$$

and by (10),

$$
\begin{aligned}
q_{k}^{N-B}\left(z_{k}(q)\right) & =f_{k}^{N-B}\left(z_{k}(q)\right) \\
& =f_{k}^{N-B}\left(\left(v_{1} \mid U_{1}, d_{e}\right), \cdots,\left(v_{k} \mid U_{k}, d_{e}\right)\right)(\text { by }(16) \text { and }(18)) \\
& =f_{k}^{N-B}\left(v_{1}, \cdots, v_{k}\right) \text { by }(\operatorname{Lemma} 3.3) \\
& =p_{k+1}^{N-B}(\text { by }(12)) .
\end{aligned}
$$

Combining (20) and (21), we obtain $q_{k}\left(z_{k}(q)\right)=p_{k+1}$.

Hence

$$
c\left(q_{k}\left(z_{k}(q)\right)\right)=p_{k+1},
$$

and it follows from this and (8) that

$$
u\left(c\left(q_{k}\left(z_{k}(q)\right)\right)\right)=u\left(p_{k+1}\right)=v_{k+1} \mid U_{k+1} .
$$

Combining (19) and (22), we obtain

$$
z_{k+1}(q) \mid U_{1} \times \cdots \times U_{k+1}=\left(v_{1}\left|U_{1}, \cdots, v_{k+1}\right| U_{k+1}\right),
$$

which completes the inductive step and the proof of (15). Hence (22) holds for all $k$, and therefore

$$
\begin{aligned}
H_{k+1}(q) & =H\left(c\left(q_{k}\left(z_{k}(q)\right)\right)\right)(\text { by } 6.6 \text { and } 6.7 \text { of }[1]) \\
& =(\psi \circ u)\left(c\left(q_{k}\left(z_{k}(q)\right)\right)\right)(\text { by } \S 6 \text { of }[1]) \\
& =\psi\left(u\left(c\left(q_{k}\left(z_{k}(q)\right)\right)\right)\right) \\
& =\psi\left(v_{k+1} \mid U_{k+1}\right)(\text { by }(22)) \\
& =H_{k+1}(v)(\text { by } 6.10 \text { of }[1]) .
\end{aligned}
$$

It then follows from 6.11 of [1] and from 3.4 that

$$
S_{k}(q)=S_{k}(v) \text {. }
$$

Applying (7), we obtain that

$$
S_{k}^{B}(q) \geq H^{B}(f)+\varepsilon^{B} \text { for infinitely many } k .
$$

In particular,

$$
\min _{i \in B}\left(S_{k}^{i}(q)-H^{i}(f)\right) \geq \min _{i \in B} \varepsilon^{i}
$$

for infinitely many $k$, and it follows that

$$
\lim _{k \rightarrow \infty} \sup _{i \in B} \min _{i}\left(S_{k}^{i}(q)-H^{i}(f)\right) \geq \min _{i \in B} \varepsilon^{i}>0 .
$$

Now by (13) and (14), $q$ is a supergame $p$-strategy vector. By (1) it satisfies 3.6 and by (23) it satisfies 3.7. Hence by $3.5, f$ cannot be 
a strong equilibrium $p$-point, a contradiction. This completes the proof of 3.11 .

\section{THEOREM 3.12. $\quad F_{p} \cap \widetilde{S_{c}}=\widetilde{S_{p}}$.}

Proof. The proof is similar to that of 3.11. It will be omitted.

The following formulae follow easily from the indicated definitions and theorems.

$$
\begin{aligned}
H\left(S_{p}\right) & \subset H\left(S_{c}\right)(\text { by } 3.11) . \\
H\left(\tilde{S}_{p}\right) & \subset H\left(\tilde{S}_{c}\right)(\text { by } 3.12) . \\
S_{p} & \subset \tilde{S}_{p}(\text { by } 3.5) \\
H\left(S_{p}\right) & \subset H\left(\tilde{S}_{p}\right)(\text { by } 3.15) .
\end{aligned}
$$

Finally, we mention the following theorem, which will be needed in the sequel.

THEOREM 3.17. A supergame p-strategy vector $f$ is summable if and only if it is summable in the mean.

Proof. The necessity follows at once from 6.9 of [1]. For sufficiency, we must show that if $f$ is summable in the mean, then a sequence of random variables distributed according to $E_{k}(f)$ obeys the strong law of large numbers. But this follows at once from 3.9.

4. Supergame pure strategies in games of perfect information. In a game $G$ of perfect information, the information that a player $i$ has about the outcome of each previous play ${ }^{2}$ may be described as follows, (4.1) He knows which terminal was reached.

(4.2) He knows which pure strategy he himself played.

Formally, let $W$ be the set of terminals in $G$, and let

$$
\lambda: P \rightarrow W
$$

be the function that associates with each pure strategy vector $p$ the terminal $\lambda(p)$ that results when $p$ is played (in the notation of [9], if $\pi \in P, \lambda(\pi)$ is the unique $w \in W$ for which $\left.p_{\pi}(w)=1\right)$. Then for each $i \in B$ and $p \in P$,

$$
u^{i}(p)=\left(\lambda(p), p^{i}\right) .
$$

If he wishes, the reader may regard 4.3 as the definition of $u^{i}$ for games of perfect information.

Actually, each player may with impunity discard the additional information obtained from 4.2 as long as he restricts himself to the use of supergame $p$-strategies. Formally, we may say that in a game of perfect information, each supergame $p$-strategy $f^{i}$ is equivalent to one

\footnotetext{
${ }^{2} \mathrm{We}$ are discussing that information that is characterized by the information function $u^{i}$.
} 
in which $f_{k}^{i}$ depends only on the $\lambda(p)$, not on the $p^{i}$. To lend meaning to this statement, we must give a suitable definition of equivalence.

DEFINITION 4.4. Two supergame p-strategies $f^{i}$ and $g^{i}$ are said to be equivalent $\left(f^{i} \simeq g^{i}\right)$ if for each supergame p-strategy $(N-i)$-vector $\theta^{N-i}$, we have

$$
H_{k}\left(f^{i}, \theta^{N-i}\right)=H_{k}\left(g^{i}, \theta^{N-i}\right), \quad k \geq 1 .
$$

COROLlaRY 4.5. Let $B \subset N$. If two supergame p-strategy vectors $f$ and $g$ are equivalent, then for each supergame p-strategy $(N-B)$ vector $\theta^{N-B}$, we have

$$
H_{k}\left(f^{B}, \theta^{N-B}\right)=H_{k}\left(g^{B}, \theta^{N-B}\right), \quad k \geq 1 .
$$

Proof. Let

$$
B=\left\{i_{1}, \cdots, i_{b}\right\} .
$$

Then since $f^{i} \simeq g^{i}$ for each $i$ it follows that for each $k \geq 1$,

$$
\begin{aligned}
H_{k}\left(f^{B}, \theta^{N-B}\right) & =H_{k}\left(g^{i_{1}},\left(f^{B-i_{1}}, \theta^{N-B}\right)\right) \\
& =H_{k}\left(g^{i_{1}}, g^{i_{2}},\left(f^{B-i_{1}-i_{2}}, \theta^{N-B}\right)\right) \\
& =H_{k}\left(\left(g^{i_{1}}, \cdots, g^{i_{b}}\right), \theta^{N-B}\right) \\
& =H_{k}\left(g^{B}, \theta^{N-B}\right) .
\end{aligned}
$$

This completes the proof.

DEFINITION 4.6. Let $G$ be a game of perfect information. A supergame $p^{*}$-strategy $f^{i}$ is a supergame p-strategy for which for each $k \geq 1$, and pair $\left(p_{1}, \cdots, p_{k}\right)$ and $\left(q_{1}, \cdots, q_{k}\right)$ of sequences of pure strategy vectors, we have

$$
\begin{array}{cl}
\lambda\left(p_{j}\right)=\lambda\left(q_{\jmath}\right), & 1 \leq j \leq k, \\
\Rightarrow \quad & f_{k}^{i}\left(\left(\left(\lambda\left(p_{1}\right), p_{1}^{i}\right), d_{e}\right), \cdots,\left(\left(\lambda\left(p_{k}\right), p_{k}^{i}\right), d_{e}\right)\right) \\
& =f_{k}^{i}\left(\left(\left(\lambda\left(q_{1}\right), q_{1}^{i}\right), d_{e}\right), \cdots,\left(\left(\lambda\left(q_{k}\right), q_{k}^{i}\right), d_{e}\right)\right) .
\end{array}
$$

For convenience, we will sometimes make use of the following conventions:

Convention 4.7. When $f^{i}$ is a supergame $p^{*}$-strategy, write

$$
f_{k}^{i}\left(\lambda\left(p_{1}\right), \cdots, \lambda\left(p_{k}\right)\right)
$$

instead of

$$
f_{k}^{i}\left(\left(\left(\lambda\left(p_{1}\right), p_{1}^{i}\right), d_{e}\right), \cdots,\left(\left(\lambda\left(p_{k}\right), p_{k}^{i}\right), d_{e}\right)\right) .
$$

Convention 4.8. When $f^{i}$ is a supergame $p$-strategy, write

$$
f_{k}^{i}\left(u^{i}\left(p_{1}\right), \cdots, u^{i}\left(p_{k}\right)\right)
$$

instead of 


$$
f_{k}^{i}\left(\left(u^{i}\left(p_{1}\right), d_{e}\right), \cdots,\left(u^{i}\left(p_{k}\right), d_{e}\right)\right) .
$$

The use of these conventions is justified by Definition 4.6 and Lemma 3.3 respectively

THEOREM 4.9. In a game $G$ of perfect information, every supergame p-strategy is equivalent to a supergame $p^{*}$-strategy.

Proof. Let $f^{i}$ be a supergame $p$-strategy in $G$. For any sequence of terminals $\left(\alpha_{1}, \cdots, \alpha_{k}\right)$ we may define $g_{k}^{i}\left(\alpha_{1}, \cdots, \alpha_{k}\right)$ by means of the following recursion:

$$
g_{0}^{i}=f_{0}^{i}
$$

( 2 ) $g_{j}^{i}\left(\alpha_{1}, \cdots, \alpha_{j}\right)=f_{j}^{i}\left(\left(\alpha_{1}, g_{0}^{i}\right),\left(\alpha_{2}, g_{1}^{i}\left(\alpha_{1}\right)\right), \cdots,\left(\alpha_{j}, g_{j-1}^{i}\left(\alpha_{1}, \cdots, \alpha_{j-1}\right)\right)\right), \quad j \leq k$.

Let $\theta^{N-i}$ be an arbitrary supergame $p$-strategy $(N-i)$-vector. We prove by induction on $k$ that

$$
z_{k}\left(f^{i}, \theta^{N-i}\right)=z_{k}\left(g^{i}, \theta^{N-i}\right),
$$

For $k=1$, (3) follows at once from (1) and 6.3 of [1]. Suppose (3) has been proved for $k \leq j$. Set

$$
\xi=\left(f^{i}, \theta^{N-i}\right),
$$$$
\zeta=\left(g^{i}, \theta^{N-i}\right) .
$$

Then by the induction hypothesis,

$$
z_{k}(\xi)=z_{k}(\zeta),
$$$$
k \leq j .
$$

Hence

$$
\begin{aligned}
\text { (6) } & g_{j}^{i}\left(z_{j}(\xi)\right)=g_{j}^{i}\left(x_{1}(\xi), x_{2}(\xi), \cdots, x_{j}(\xi)\right) \quad(\text { by } 3.2) \\
= & g_{j}^{i}\left(x_{1}(\xi)\left|W, x_{2}(\xi)\right| W, \cdots, x_{j}(\xi) \mid W\right) \quad(\text { by } 4.7) \\
= & f_{J}^{i}\left(\left(x_{1}(\xi) \mid W, g_{0}^{i}\right),\left(x_{2}(\xi) \mid W, g_{1}^{i}\left(z_{1}(\xi)\right)\right), \cdots,\left(x_{j}(\xi) \mid W, g_{j-1}^{i}\left(z_{j-1}(\xi)\right)\right)\right) \quad(\text { by }(2)) \\
= & f_{J}^{i}\left(\left(x_{1}(\xi) \mid W, g_{0}^{i}\right),\left(x_{2}(\xi) \mid W, g_{1}^{i}\left(z_{1}(\xi)\right)\right), \cdots,\left(x_{j}(\xi) \mid W, g_{j-1}^{i}\left(z_{j-1}(\zeta)\right)\right)\right)(\text { by }(5)) .
\end{aligned}
$$

Now by 6.2 and 6.5 of [1], we have

$$
\begin{aligned}
x_{k}(\zeta) & =s\left(\zeta_{k-1}\left(z_{k-1}(\zeta)\right)\right) \\
& =\left(u\left(c\left(\zeta_{k-1}\left(z_{k-1}(\zeta)\right)\right)\right), d\left(\zeta_{k-1}\left(z_{k-1}(\zeta)\right)\right)\right) .
\end{aligned}
$$

But by 2.7 of [1] and by 3.2 ,

$$
c\left(\zeta_{k-1}\left(z_{k-1}(\zeta)\right)\right)=\zeta_{k-1}\left(z_{k-1}(\zeta)\right)
$$

Applying this to (7), we obtain

$$
\begin{aligned}
& x_{k}^{i}(\zeta) \mid U_{k}^{i}=u^{i}\left(\zeta_{k-1}\left(z_{k-1}(\zeta)\right)\right) \\
& =\left(\lambda\left(\zeta_{k-1}\left(z_{k-1}(\zeta)\right)\right), \zeta_{k-1}^{i}\left(z_{k-1}(\zeta)\right)\right) \\
& =\left(\lambda\left(\zeta_{k-1}\left(z_{k-1}(\zeta)\right)\right), g_{k-1}^{i}\left(z_{k-1}(\zeta)\right)\right) \quad(\text { by }(4)) \text {. }
\end{aligned}
$$


Hence

$$
x_{k}^{i}(\zeta) \mid P^{i}=g_{k-1}^{i}\left(z_{k-1}(\zeta)\right),
$$

Applying this to (6), we obtain

$$
\begin{aligned}
g_{j}^{i}\left(z_{j}(\xi)\right) & =f_{j}^{i}\left(\left(x_{1}(\xi)\left|W, x_{j}^{i}(\xi)\right| P^{i}\right), \cdots,\left(x_{j}(\xi)\left|W, x_{1}^{i}(\xi)\right| P^{i}\right)\right) \\
& =f_{j}^{i}\left(\left(x_{1}(\xi)\left|W, x_{j}^{i}(\xi)\right| P^{i}\right), \cdots,\left(x_{j}(\xi)\left|W, x_{1}^{i}(\xi)\right| P^{i}\right)\right) \\
& =f_{j}^{i}\left(x_{1},(\xi)\left|U_{1}^{i}, \cdots, x_{j}(\xi)\right| U_{k}^{i}\right) \quad(\text { by } 4.3) \\
& =f_{j}^{i}\left(x_{1}(\xi), \cdots x_{j}(\xi)\right) \quad(\text { by } 4.8) \\
& =f_{j}^{i}\left(z_{j}(\xi)\right) .
\end{aligned}
$$

Applying (4), we obtain

$$
\xi_{j}\left(z_{j}(\xi)\right)=\zeta_{j}\left(z_{j}(\xi)\right)
$$

Hence

$$
\begin{array}{rlrl}
x_{j+1}(\zeta) \mid U_{j+1} & & \\
& =u\left(\zeta_{j}\left(z_{j}(\zeta)\right)\right) & & (\text { by } 6.5 \text { of }\lceil\mathbf{1}]) \\
& =u\left(\zeta_{j}\left(z_{j}(\xi)\right)\right) & & (\text { by }(5)) \\
& =u\left(\xi_{j}\left(z_{j}(\xi)\right)\right) & & (\text { by }(8)) \\
& =x_{j+1}(\xi) \mid U_{j+1} . &
\end{array}
$$

Hence by 3.2 ,

$$
z_{j+1}(\zeta)\left|U_{1} \times \cdots \times U_{j+1}=z_{j+1}(\xi)\right| U_{1} \times \cdots \times U_{j+1},
$$

and since

$$
z_{j+1}(\zeta)\left|R_{1} \times \cdots \times R_{j+1}=d_{e} \times \cdots \times d_{e}=z_{j+1}(\xi)\right| R_{1} \times \cdots \times R_{j+1},
$$

we conclude from (9) that

$$
z_{j+1}(\zeta)=z_{j+1}(\xi)
$$

This completes the induction and the proof of (3). Applying 6.6 and 6.7 of [1] to (3), we obtain

$$
H_{k}\left(f^{i}, \theta^{N-i}\right)=H_{k}\left(g^{i}, \theta^{N-i}\right), \quad k \geq 1 .
$$

Hence by 4.4 ,

$$
f^{i} \simeq g^{i} \text {. }
$$

But $g^{i}$ is by its definition a supergame $p^{*}$-strategy, and thus our proof is completed.

Parallel to Definition 3.5, we may make the following definition:

Definition 4.10. A strong equilibrium $p^{*}$-point $f$ is a summable supergame $p^{*}$-strategy vector for which there is no $B \subset N$ and supergame $p^{*}$-strategy vector $g$ satisfying 3.6 and 3.7 . 
The set of all strong equilibrium $p^{*}$-points is denoted by $S_{p^{*}}$. If 3.7 is replaced by 3.8 , the resulting set of points is denoted by $\widetilde{S}_{p^{*}}$.

If we can succeed in restricting our considerations to supergame $p^{*}$-strategies then we will have considerably simplified our problem, because then the information available about previous plays is the same for all players (so that the information function may be regarded as 1-dimensional rather than $n$-dimensional). That we may without loss of generality restrict ourselves in this way is the content of the next theorem.

THEOREM 4.11. In a game $C$ of perfect information, a summable supergame p-strategy vector $f$ is a strong equilibrium p-point if there is a strong equilibrium $p^{*}$-point $f_{*}$ equivalent to $f$.

Proof. Suppose

$$
f \notin S_{p} \text {. }
$$

Then there is a $B \subset N$ and a supergame $p$-strategy vector $g$ satisfying 3.6 and 3.7. In accordance with 4.9 , there is a supergame $p^{*}$-strategy $B$-vector $g_{*}^{B}$ for which

$$
g_{*}^{B} \simeq g^{B}
$$

Define

$$
g_{*}^{N-B}=f_{*}^{N-B}
$$

By hypothesis we have

$$
f_{*}^{N-B} \simeq f^{N-B} .
$$

Combining (1), (2), (3), and 3.6, we obtain

$$
g_{*} \simeq g .
$$

From (4), 3.4 and 4.5 it follows that

$$
S_{k}(g)=S_{k}\left(g_{*}\right)
$$

for each $k$. By hypothesis,

$$
f \simeq f_{*} \cdot
$$

Applying 6.8 of [1] and 4.5 to (6), we obtain

$$
H(f)=H\left(f_{*}\right) \text {. }
$$

From 3.7, (5), and (7) it follows that

$$
\limsup _{k \rightarrow \infty} \min _{i \in B}\left(S_{k}^{i}\left(g_{*}\right)-H^{i}\left(f_{*}\right)\right)>0 .
$$

From (2), (8), and 4.10 it follows that $f_{*} \notin S_{p^{*}}$, which contradicts the hypothesis. This completes the proof.

Corollary 4.12. $H\left(S_{p^{*}}\right) \subset H\left(S_{p}\right)$. 
Proof. Follows from 6.8 of [1], 4.5, and 4.11.

The following theorems (4.13 through 4.16) will not be used in the sequel; they are included for the sake of completeness. The proofs use the same ideas as those already, given, and will be omitted.

THeORem 4.13. Conversely to 4.11, a summable supergame p-strategy vector $f$ is a strong equilibrium p-point only if there is a strong equilibrium $p^{*}$-point $f_{*}$ equivalent to $f$.

CoROLlaRY 4.14. $H\left(S_{p}\right)=H\left(S_{p^{*}}\right)$.

TheOREM 4.15. $S_{p^{*}} \subset \widetilde{S}_{p^{*}}$

THEOREM 4.16. $H\left(\tilde{S_{p}}\right)=H\left(\tilde{S_{p^{*}}}\right)$.

Theorems analogous to 4.11 and 4.13 for $S_{p^{*}}$ may also be proved.

For supergame $p^{*}$-strategy vectors $f$, formulas 6.3 through 6.7 of [1] may be rewritten as follows:

$$
\begin{aligned}
z_{1} & =\lambda\left(f_{0}\right) \\
z_{k} & =\left(z_{k-1}, x_{k}\right) \\
x_{k} & =\lambda\left(f_{k-1}\left(z_{k-1}\right)\right) \\
E_{k} & =E\left(f_{k-1}\left(z_{k-1}\right)\right) \\
H_{k} & =H\left(E_{k}\right) .
\end{aligned}
$$

Here we are making use of the notation introduced in convention 4.7.

5. The main theorem. We make use of two lemmas. The first tells us that at an acceptable point in a game of perfect information, $N-B$ can always retaliate for a defection by $B$ by means of a single pure strategy. The second tells us that any payoff that can be obtained by a $c$-strategy vector in $G$ can also be obtained by a supergame $p$-strategy vector (or even by a supergame $p^{*}$-strategy vector).

LemMa 5.1. Let $G$ be a game of perfect information. Let $B \subset N$, and let $h$ be a vector. If there is a $c^{N-B} \in C^{N-B}$ such that for all $c^{B} \in C^{B}$, there is an $i \in B$ for which

$$
H^{i}\left(c^{B}, c^{N-B}\right) \leq h^{i},
$$

then there is a $p^{N-B} \in P^{N-B}$ such that for all $c^{B} \in C^{B}$, there is an $i \in B$ for which 


$$
H^{i}\left(c^{B}, p^{N-B}\right) \leq h^{i}
$$

Proof. $H^{B}\left(C^{B}, c^{N-B}\right)$ is easily seen to be a convex subset of the euclidean $B$-space $R^{B}$. By the hypothesis of the lemma, $H^{B}\left(C^{B}, c^{N-B}\right)$ cannot intersect the open "corner" or "octant" in $R^{B}$ given by the inequalities

$$
x^{i}>h^{i},
$$

This "corner" is also convex. Applying the separation theorem for convex sets $^{3}$, we obtain a hyperplane

$$
\sum_{i \in B} a^{i} x^{i}=k
$$

which passes through $h^{B}$, and which separates $H^{B}\left(C^{B}, c^{N-B}\right)$ from the "corner" given by (3). In other words, we have

$$
\sum_{i \in B} a^{i} h^{i}=k,
$$

and we may assume without loss of generality that

$$
\sum_{i \in B} a^{i} H^{i}\left(c^{B}, c^{N-B}\right) \leq k \quad \text { for } c^{B} \in C^{B}
$$

and

$$
\sum_{i \in B} a^{i} x^{i}>k \text { for } x^{B} \text { satisfying (3). }
$$

(if the inequalities (6) and (7) are reversed, then we may obtain them in the given form by multiplying both sides of (4) by -1). From (3) and (7) it follows that

$$
a^{B} \geq 0 \text {. }
$$

Since (4) defines a hyperplane, there must also be an $i \in B$ for which

$$
a^{i} \neq 0 \text {. }
$$

Define a two-person, zero-sum game $G_{*}$ as follows: There are two players, 1 and 2. The game tree of $G_{*}$ is the same as that of $G$, and $G_{*}$ is also a game of perfect information. Player 1 has all the moves that members of $B$ have in $C$, and player 2 has all the moves that members of $N-B$ have in $G$. Thus the mixed strategy space of player 1 is $C^{B}$, and the mixed strategy space of player 2 is $C^{N-B}$ (we will also use the notation $M^{1}$ and $M^{2}$ for these mixed strategy spaces). The payoff in $G_{*}$ will be denoted by $H_{*}$; it is defined by

$$
H_{*}^{1}\left(p^{B}, p^{N-B}\right)=\sum_{i \in B} a^{i} H^{i}\left(p^{B}, p^{N-B}\right)
$$

3 See for instance [10], pp. 29 and 81. 


$$
H_{*}^{2}=-H_{*}^{1}
$$

From (10) it follows that

$$
H_{*}^{1}\left(c^{B}, c^{N-B}\right)=\sum_{i \in B} a^{i} H^{i}\left(c^{B}, c^{N-B}\right)
$$

for all $c^{B} \in C^{B}$. Combining (12) with (6) and the hypothesis of the lemma, we obtain the existence of a $c^{N-B} \in C^{N-B}$ such that for all $c^{B} \in C^{B}$, we have

$$
H_{*}^{1}\left(c^{B}, c^{N-B}\right) \leq k
$$

Restated in terms of mixed strategies in $C_{*}$, we have the existence of a mixed strategy $m_{*}^{2} \in M_{*}^{2}$ (namely $\left.c^{N-B}\right)$, such that for all $m_{*}^{1} \in M_{*}^{1}$, we have

$$
H_{*}^{1}\left(m_{*}^{1}, m_{*}^{2}\right) \leq k
$$

By (11), $G_{*}$ is zero-sum as well as two person. (13) merely tells us that

$$
v\left(G_{*}\right) \leq k,
$$

where $v\left(G_{*}\right)$ denotes the value of $G_{*}$. By the theorem of von Neumann on two-person zero-sum games of perfect information, we have the existence of an optimal pure strategy for player 2 in $G_{*}$. Hence there is a $p_{*}^{2} \in p_{*}^{2}$ (i.e. a $p^{N-B} \in P^{N-B}$ ) such that for all $m_{*}^{1} \in M_{*}^{1}$ (i.e. for all $\left.c^{B} \in C^{B}\right)$, we have

$$
H_{*}^{1}\left(m_{*}^{1}, p_{*}^{2}\right) \leq v\left(G_{*}\right)
$$

(i.e., by $(10)$,

$$
\left.\sum_{i \in B} a^{i} H^{i}\left(c^{B}, p^{N-B}\right) \leq v\left(G_{*}\right)\right)
$$

Combining (5), (14), and (15), we obtain

$$
\sum_{i \in B} a^{i} H^{i}\left(c^{B}, p^{N-B}\right) \leq \sum_{i \in B} a^{i} h^{i}
$$

for all $c^{B} \in C^{B}$.

From (16) it follows that

$$
\sum_{i \in B} a^{i}\left(H^{i}\left(c^{B}, p^{N-B}\right)-h^{i}\right) \leq 0
$$

for all $c^{B} \in C^{B}$. Combining this with (8) and (9), we obtain for each $c^{B} \in C^{B}$, the existence of at least one $i \in B$ for which

$$
H^{i}\left(c^{B}, p^{N-B}\right)-h^{i} \leq 0
$$

This completes the proof of the lemma. 
The next lemma tells us that the non-negative integers can be partitioned into disjoint subsets whose asymptotic densities will yield an arbitrary finite set of non-negative real numbers adding up to 1 .

Lemma 5.2. Let $Z$ be a finite set, and let $y \in C(Z)$. For any mapping $\pi$ from the set $K$ of all non-negative integers into $Z$, and for any $k \in K$ and $z \in Z$, let $\rho_{\pi}(k ; z)$ denote the number of $j \in K$ for which

$$
j \leq k
$$

and

$$
\pi(j)=z
$$

Then there is a $\pi$ for which

$$
\lim _{k \rightarrow \infty} \frac{\rho_{\pi}(k ; z)}{k+1}=y(z)
$$

for all $z \in Z$.

$\pi(j)$ will also be denoted by $\pi(j ; y)$, and $\rho_{\pi}(k, z)$ by $\rho(k ; z ; y)$.

The proof is not difficult. It will be omitted.

THEOREM 5.3. In a game $G$ of perfect information,

$$
H\left(A_{c}\right) \subset H\left(S_{p^{*}}\right) .
$$

Proof. In its main outlines, the proof is analogous to that of Theorem 3 of [1], which states that $H\left(A_{c}\right) \subset H\left(S_{c}\right)$. The details, however, differ considerably in the two cases. Both proofs are divided into three parts: Given an acceptable payoff vector $h$, we must first find a sequence of strategy vectors which will yield a payoff of $h$ in the supergame (under the assumption that the players are all "loyal"). Next, we must find a way to determine which players, if any, are disloyal; and finally, we must find a way to punish the disloyal players. All these elements must be incorporated into a supergame strategy vector. In Theorem 3 of [1], the first of these tasks was accomplished by having the players play the same $c$-strategy vector on each play, namely the one that yields an expected payoff of $h$. Here this cannot be done, because the players must restrict themselves to pure strategies on each play. They must therefore play different pure strategy vectors on different plays in such a way so that the limiting payoff is $h$; to show that this can be done, use must be made of Lemma 5.2. As for the second task, this was accomplished in Theorem 3 of [1] by simply noting the make-up of the coalitions; here this cannot be done, because in supergame $p^{*}$-strategy vectors, there are no coalitions. Instead, use 
must be made of the perfect information that each player has. Finally, a group $B$ of disloyal players could be punished in Theorem 3 of [1] by use of the $c$-strategy $(N-B)$-vector $c^{N-B}$ provided in the definition of acceptability; here only pure strategy $(N-B)$-vectors may be used, so that recourse must be had to Lemma 5.1. For a more detailed intuitive statement of the proof, see $\S 10$ of [1].

We now give the detailed proof of 5.3.

Let $h \in H\left(A_{c}\right)$, and suppose $\gamma^{N} \in A_{c}$ is such that

$$
H\left(\gamma^{N}\right)=h \text {. }
$$

Then by 4.3 of [1], for each $B \subset N$ there is a $c^{N-B} \in C^{N-B}$, such that for each $c^{B} \in C^{B}$, there is an $i \in B$ for which

$$
H^{i}\left(c^{B}, c^{N-B}\right) \leq h^{i} .
$$

Applying Lemma 5.1, we obtain for each $B \subset N$ a pure strategy $(N-B)$ vector $\gamma^{N-B}$, such that for each $c^{B} \in C^{B}$, there is an $i \in B$ for which

$$
H^{i}\left(c^{B}, \gamma^{N-B}\right) \leq h^{i} .
$$

For each $j \geq 1$, let $W_{j}$ be a copy of $W$. $W_{j}$ represents the set of possible outcomes of the $j$ th play. Let

$$
Q_{k}=W_{1} \times \cdots \times W_{k} ;
$$

$Q_{k}$ represents the set of possible outcomes for the first $k$ plays, and as such is the domain of the function $f_{k}^{i}$.

Let $g$ be any supergame $p^{*}$-strategy vector in $G$. We define a compliance function $\alpha\left(v_{1}, \cdots, v_{k} ; g\right)$ for all $\left(v_{1}, \cdots, v_{k}\right) \in Q_{k}$ as follows: which

Definition (3). $\alpha\left(v_{1}, \cdots, v_{k} ; g\right)$ is the maximal subset $A$ of $N$ for

$$
v_{j} \in \lambda\left(g_{j-1}^{A}\left(v_{1}, \cdots, v_{j-1}\right) \times P^{N-A}\right) \quad \text { for } j=1, \cdots, k .
$$

For each member of $Q_{k}$, $\alpha$ tells which subset of $N$ has been "loyal" to, or has complied with, the supergame $p^{*}$-strategy vector $g$.

It is not difficult to see that for each $g$, we have

$$
\alpha\left(z_{k}(g) ; g\right)=N
$$

for $k \geq 1$.

To show (4), it is sufficient to show that $N$ is the maximal set satisfying (3), i.e. that we have

$$
\begin{aligned}
x_{j}(g) & =\lambda\left(g_{j-1}^{N}\left(z_{j-1}(g)\right) \times P^{N-N}\right), & & j=1, \cdots, k \\
& =\lambda\left(g_{j-1}\left(z_{j-1}(g)\right)\right), & & j=1, \cdots, k .
\end{aligned}
$$

But this follows at once from 4.19 . 
Moreover, it follows from (3) that

$$
\alpha=N \text { when } k=0 \text {. }
$$

We are now ready to define a strong equilibrium $p^{*}$-point whose payoff is $h$.

For $k \geq 0$ and $q_{k} \in Q_{k}$, define

$$
\left\{\begin{array}{ll}
f_{k}\left(q_{k}\right)=\pi\left(k ; \gamma^{N}\right), & \text { if } \alpha\left(q_{k} ; f\right)=N \\
f_{k}^{\alpha\left(q_{k} ; f\right)}\left(q_{k}\right)=\gamma^{\alpha\left(q_{k} ; f\right)} \\
f_{k}^{N-\alpha\left(q_{k} ; f\right)}\left(q_{k}\right)=\text { arbitrary }
\end{array}\right\} \text { otherwise }
$$

Definition (6) is a recursive definition; $\alpha\left(q_{k} ; f\right)$ depends only on $f_{0}, \cdots, f_{k-1}$, not on $f_{k}$.

Set $z_{k}=z_{k}(f)$ for $k \geq 1$. We first prove

$$
f_{k}\left(z_{k}\right)=\pi\left(k ; \gamma^{N}\right) \text { for } k \geq 0 .
$$

For $k>0$, (7) follows from (6) and (4); for $k=0$, it follows from (6) and (5).

Combining (7) with 4.20 and 4.21 , we obtain

$$
H_{k+1}(f)=H\left(\pi\left(k ; \gamma^{N}\right)\right) \text { for } k \geq 0 \text {. }
$$

Hence

$$
\begin{aligned}
\sum_{r=1}^{k+1} H_{r}(f) & =\sum_{r=0}^{k} H_{r+1}(f) \\
& =\sum_{r=0}^{k} H\left(\pi\left(r ; \gamma^{N}\right)\right) \quad(\text { by }(8)) \\
& =\sum_{y \in P} \rho\left(k ; y ; \gamma^{N}\right) H(y) \quad(\text { by } 5.2) .
\end{aligned}
$$

Hence

$$
\begin{aligned}
\lim _{k \rightarrow \infty} \frac{1}{k} \sum_{r=1}^{k} H_{r}(f) & =\lim _{k \rightarrow \infty} \frac{1}{k+1} \sum_{r=1}^{k+1} H_{r}(f) \\
& =\lim _{k \rightarrow \infty} \frac{1}{k+1} \sum_{y \in P} \rho\left(k ; y ; \gamma^{N}\right) H(y) \\
& =\sum_{y \in P} H(y) \lim _{k \rightarrow \infty} \frac{1}{k+1} \rho\left(k ; y ; \gamma^{N}\right) \\
& =\sum_{y \in P} \gamma^{N}(y) H(y) \quad(\text { by } 5.2) \\
& =H\left(\gamma^{N}\right) \\
& =h \quad(\text { by }(1)) .
\end{aligned}
$$

Applying 6.8 of [1], we obtain 


$$
H(f)=h .
$$

By $3.17, f$ is also summable.

It remains to prove that $f$ is a strong equilibrium $p^{*}$-point. Suppose not. Then there is a $B \subset N$ and a supergame $p^{*}$-strategy vector $g$ satisfying 3.6 and 3.7. We must then have

LEMma (10). $\alpha\left(z_{k}(g) ; f\right)$ is monotone decreasing with $k$.

Proof. By 4.18,

$$
z_{k}(g)=\left(x_{1}(g), \cdots, x_{k}(g)\right) .
$$

The result now follows from (3).

From 4.19 we obtain

$$
\begin{aligned}
x_{j}(g)= & \lambda\left(g_{j-1}\left(z_{j-1}(g)\right)\right) \\
= & \lambda\left(g_{j-1}^{N-B}\left(z_{j-1}(g)\right), g_{j-1}^{B}\left(z_{j-1}(g)\right)\right) \\
& \in \lambda\left(g_{j-1}^{N-B}\left(z_{j-1}(g)\right) \times P^{B}\right) \\
= & \lambda\left(f_{j-1}^{N-B}\left(z_{j-1}(g)\right) \times P^{B}\right) \quad(\text { by } 3.6) \\
= & \lambda\left(f_{j-1}^{N-B}\left(x_{1}(g), \cdots, x_{j-1}(g)\right) \times P^{B}\right) \quad(\text { by } 4.18) .
\end{aligned}
$$

It now follows from (3) that

$$
N-B \subset \alpha\left(z_{k}(g) ; f\right) \text { for } k \geq 1 .
$$

Combining (11) with (5), we obtain

$$
N-B \subset \alpha\left(z_{k}(g) ; f\right) \text { for } k \geq 0 .
$$

From (10) we obtain the existence of a set $B(g) \subset N$ and a non-negative integer $k_{0}$ such that

$$
\alpha\left(z_{k}(g) ; f\right)=N-B(g) \text { for } k \geq k_{0} .
$$

Combining (12) and (13), we obtain

$$
B(g) \subset B
$$

If $B(g)=\phi$, then from (13) we obtain

$$
\alpha\left(z_{k}(g) ; f\right)=N \text { for } k \geq k_{0},
$$

whence, using (10), we deduce that

$$
\alpha\left(z_{k}(g) ; f\right)=N \text { for } k \geq 0 .
$$

Using (3) and 4.18, we deduce from (15) that

$$
x_{k}(g)=\lambda\left(f_{k-1}\left(z_{k-1}(g)\right)\right) \text { for } k \geq 1 .
$$


From (16) and 4.17 we deduce

$$
x_{1}(g)=\lambda\left(f_{0}\right)=x_{1}(f),
$$

and a simple inductive argument based on (16), 4.18 and 4.19 leads to the conclusion that

$$
z_{k}(g)=z_{k}(f) \text { for } k \geq 1 .
$$

Applying 4.20 and 4.21 , we obtain

$$
H_{k}(g)=H_{k}(f) \text { for } k \geq 1 \text {. }
$$

From 6.8 of [1], 3.4, and (17) it follows that

$$
\lim _{k \rightarrow \infty} S_{k}(g)=H(f),
$$

which contradicts 3.6. Thus the assumption $B(g)=\phi$ has led to a contradiction, and we may conclude that

$$
B(g) \neq \phi \text {. }
$$

Combining (6), (13), and (18), we obtain

$$
f_{k}^{N-B(g)}\left(z_{k}(g)\right)=\gamma^{N-B(g)} \text { for } k \geq k_{0} .
$$

Let $\mu$ be the payoff function defined on $W$, so that

$$
H=\mu \circ \lambda \text {. }
$$

Our $\mu$ is what is called $h$ in [3]; it may also be defined by

$$
\mu=\psi \mid W \text {, }
$$

where $\psi$ is as in $\S 6$ of $[1]$. We then have

$$
\begin{aligned}
H_{k}(g) & \left.=H\left(E_{k}(g)\right) \quad \text { (by } 4.21\right) \\
& \left.=H\left(g_{k-1}\left(z_{k-1}(g)\right)\right) \quad \text { (by } 4.20\right) \\
& \left.=\mu\left(\lambda\left(g_{k-1}\left(z_{k-1}(g)\right)\right)\right) \quad \text { (by }(20)\right) \\
& \left.=\mu\left(x_{k}(g)\right) \quad \text { (by } 4.19\right) .
\end{aligned}
$$

Now by (3), (13), and 4.19 , we have

$$
x_{k}(g)=\lambda\left(f_{k-1}^{N-B(g)}\left(z_{k-1}(g)\right), p_{k}^{B(g)}\right),
$$

where $p_{r}^{B(g)}$ is some member of $P^{B(g)}$.

Hence for $k>k_{0}$, we have

$$
\begin{aligned}
H_{k}(g) & =\mu\left(x_{k}(g)\right) \quad(\text { by }(21)) \\
& \left.=(\mu \circ \lambda)\left(f_{k-1}^{N-B(g)}\left(z_{k-1}(g)\right), p_{k}^{B(g)}\right) \quad \text { (by }(22)\right) \\
& \left.=H\left(\gamma^{N-B(g)}, p_{k}^{B(g)}\right) \quad \text { (by }(20) \text { and }(19)\right) .
\end{aligned}
$$


Hence for $k>k_{0}$, we have by the linearity of $H$ that

$$
\begin{aligned}
\frac{1}{k-k_{0}} \sum_{r=k_{0}+1}^{k} H_{r}(g) & =\frac{1}{k-k_{0}} \sum_{r=k_{0}+1}^{k} H\left(\gamma^{N-B(g)}, p_{r}^{B(g)}\right) \\
& =H\left(\gamma^{N-B(g)}, \sum_{r=k_{0}}^{k+1} \frac{1}{k-k_{0}} p_{r}^{B(g)}\right)
\end{aligned}
$$

Applying (2), we obtain the existence of an $i \in B(g)$ such that

$$
H^{i}\left(\gamma^{N-B(g)}, \sum_{r=k_{0}}^{k+1} \frac{1}{k-k_{0}} p_{r}^{B(g)}\right)-h^{i} \leq 0 .
$$

Combining (23) and (24), we deduce that

$$
\min _{i \in B(g)}\left(\left(\frac{1}{k-k_{0}} \sum_{r=k_{0}+1}^{k} H_{r}^{i}(g)\right)-h^{i}\right) \leq 0 ;
$$

from this and (14) it follows what

$$
\min _{i \in B}\left(\left(\frac{1}{k-k_{0}} \sum_{r=k_{0}+1}^{k} H_{r}^{i}(g)\right)-h^{i}\right) \leq 0 .
$$

Now it follows easily from the boundedness of $H$ that as $k \rightarrow \infty$,

$$
\begin{aligned}
\frac{1}{k-k_{0}} \sum_{r=k_{0}+1}^{k} H_{r}^{i}(g) & =\frac{1}{k} \sum_{j=1}^{k} H_{j}^{i}(g)+O\left(\frac{1}{k}\right) \\
& =S_{k}^{i}(g)+o(1) \quad(\text { by 3.4). }
\end{aligned}
$$

Applying this to (25), we obtain that as $k \rightarrow \infty$,

$$
\min _{i \in B}\left(S_{k}^{i}(g)-h^{i}\right) \leq o(1),
$$

whence

$$
\lim _{k \rightarrow \infty} \sup \min _{i \in B}\left(S_{k}^{i}(g)-h^{i}\right) \leq 0 .
$$

Applying (9), we see that (26) contradicts 3.7. This completes the proof of 5.3 .

THEOREM 5.4. In a game $G$ of perfect information,

$$
H\left(A_{c}\right)=H\left(S_{p}\right)=H\left(\widetilde{S}_{p}\right) .
$$

In particular, $h$ is a c-acceptable payoff vector in $G$, if and only if there is a strong equilibrium c-point $f$ in supergame pure strategies for which

$$
H(f)=h .
$$


Proof. We have

$$
\begin{aligned}
H\left(A_{c}\right) & \subset H\left(S_{p^{*}}\right) & & (\text { by } 5.3) \\
& \subset H\left(S_{p}\right) & & (\text { by } 4.12) \\
& \subset H\left(S_{c}\right) & & (\text { by } 3.13) \\
& =H\left(A_{c}\right) & & (\text { by Corollary } 4 \text { of }[1])
\end{aligned}
$$

Hence equality must hold throughout, and in particular,

$$
H\left(A_{c}\right)=H\left(S_{p}\right)
$$

Next, we have

$$
\begin{array}{rlrl}
H\left(S_{p}\right) \subset H\left(\widetilde{S}_{p}\right) & & (\text { by } 3.16) \\
& \subset H\left(\widetilde{S}_{c}\right) & & (\text { by } 3.12) \\
& =H\left(A_{c}\right) & & (\text { by Corollary } 4 \text { of }[\mathbf{1}]) \\
& =H\left(S_{p}\right) & & (\text { by }(1)) .
\end{array}
$$

Hence equality must hold throughout, and we deduce

$$
H\left(S_{p}\right)=H\left(\tilde{S}_{p}\right) \text {. }
$$

(1) and (2) yield the first part of 5.4. The second part follows at once from 3.11 and the first part.

COROLlaRY 5.5. Every stable game of perfect information has strong equilibrium c-points in supergame pure strategies.

6. The converse of the main theorem. For two-person zero-sum games not involving chance, Von Neumann's theorem is known to "characterize" games of perfect information (see [4]). More precisely, if $\Gamma$ is a game structure of the above type which has the property that every game that can be obtained from $\Gamma$ (by adjunction of a payoff function $\mu$ ) has optimal pure strategies, then $\Gamma$ must be equivalent to a game structure of perfect information. What can be said in this regard for the theory presented in the previous sections?

For one thing, it is of interest to know that there are some games that do not satisfy our main theorem (Theorem 5.4). Indeed, "matching pennies" is such a game.

This game is given by

$$
\begin{aligned}
& N=(1,2) \\
& P^{1}=\left(p_{1}^{1}, p_{2}^{1}\right) \\
& P^{2}=\left(p_{1}^{2}, p_{2}^{2}\right)
\end{aligned}
$$

4 That is, every game that has any $c$-acceptable points (or, equivalently, any strong equilibrium $c$-points). See $\& 11$ of $[\mathbf{1}]$. 


$$
\begin{aligned}
H^{1}\left(p_{i}^{1}, p_{j}^{2}\right) & =\left\{\begin{aligned}
1 & \text { if } i=j \\
-1 & \text { if } i \neq j
\end{aligned}\right. \\
H^{2}(p) & =-H^{1}(p) \\
u(p) & =p .
\end{aligned}
$$

It is a two-person zero-sum game with value 0 ; hence by Theorem 1 of [1], we have

$$
H\left(A_{c}\right)=(0,0) .
$$

If 5.4 holds for this game, then it follows that

$$
H\left(S_{p}\right)=(0,0),
$$

and in particular, there is a summable strong equilibrium $p$-point $f$ such that

$$
H(f)=(0,0) .
$$

Define a supergame $p$-strategy vector $g$ by

$$
g^{2}=f^{2}
$$

and

$$
g_{k}^{1}\left(v_{1}, \cdots, v_{k}\right)=p_{i}^{1}, \text { for } k \geq 0,\left(v_{1}, \cdots, v_{k}\right) \in J_{1} \times \cdots \times J_{k},
$$

where $i$ is such that

$$
f_{k}^{2}\left(v_{1}, \cdots, v_{k}\right)=p_{i}^{2} .
$$

It is then easily seen that

$$
H_{k}^{1}(g)=1 \quad \text { for } k \geq 0,
$$

whence it follows that

$$
S_{k}^{1}(g)=1 \quad \text { for } k \geq 0 .
$$

Combining this with (1), we see that $g$ satisfies 3.7 for $B=(1) . \quad$ By (2), $g$ satisfies 3.6 for $B=(1)$. Hence $f$ cannot be a strong equilibrium $p$ point.

The above example constitutes a formalization of the familiar argument that states that no "scheme" for playing a long sequence of pennymatchings that involves only pure strategies can be optimal.

The general statement of the converse would be as follows:

CONJECTURE. Let $\Gamma$ be a game structure and suppose that every stable game that is obtained from $\Gamma$ by adjunction of a payoff function $\mu$ has a strong equilibrium p-point. Then $\Gamma$ is essentially equivalent (in the sense of [4]) to a game structure of perfect information. 
There is little doubt in my mind that this conjecture is true, if not in the given form, then at least in some other closely allied form.

7. Notation for non-cooperative games. We will make use of the notion of mixed strategies. Formally, the space $M^{i}$ of mixed strategies of player $i$ is defined to be identical with $C^{i}$. If $B$ is a subset of $N$, then we define

$$
M^{B}=\prod_{i \in B} M^{i}
$$

the cartesian product is meant. It follows that

$$
M^{B} \subset C^{B}
$$

the opposite inequality is generally false. The prefix $m$ - is an abbreviation for "mixed". The definitions relating to payoff remain unchanged.

8. Acceptable points for non-cooperative games. The non-cooperative game differs from the cooperative game chiefly in that the use of correlated strategy vectors that are not also mixed strategy vectors is forbidden. The definition of acceptability for non-cooperative games will therefore be the same as that for cooperative games (see [1], section 4), except that correlated strategy vectors must be replaced throughout by mixed strategy vectors. The intuitive reasoning behind the definition remains unchanged. It might be objected that the "concerted action" that is necessary to prevent a set of players $B$ from obtaining a payoff that is higher than at an acceptable point, is forbidden under non-cooperative rules. In fact, such concerted action will probably arise anyway as part of a "silent gentlemen's agreement" among the players of $N-B$. The only restriction is that though the players may "cooperate" in this sense (indeed, they cannot be prevented from so doing), they may not correlate their mixed strategies before a play.

Further intuitive discussion of the notion of $m$-acceptability will be found in a subsequent paper, devoted exclusively to acceptable points in non-cooperative games.

The formal definitions are as follows:

DEFINITION 8.1. Let $m_{0} \in M . \quad m_{0}$ is said to be m-acceptable if there is no $B \subset N$ such that for each $m^{N-B} \in M^{N-B}$, there is an $m^{B} \in M^{B}$ for which

$$
H^{B}\left(m^{B}, m^{N-B}\right)>H^{B}\left(m_{0}\right) .
$$

The set of all $m$-acceptable $m$-strategy vectors is denoted by $A_{m}$. Like $c$-acceptability $m$-acceptability is a "global" notion (see [1], §4). 
Definition 8.2. A payoff vector $h$ is said to be m-acceptable, if for some $m \in A_{m}$, we have

$$
H(m)=h .
$$

The following is a trivial restatement of 8.2:

Theorem 8.3. A payoff vector $h$ is $m$-acceptable if and only if for each $B \subset N$, there is an $m^{N-B} \in M^{N-B}$, such that for all $m^{B} \subset M^{B}$, there is an $i \in B$ for which

$$
H^{i}\left(m^{B}, m^{N-B}\right) \leq h^{i} .
$$

We remark that as in the cooperative case, all two-person games have $m$-acceptable points. When we go beyond two-person games we find games that have no $m$-acceptable points. The example given in $\S 11$ of [1] holds for the non-cooperative case as well, as does the intuitive discussion following the example.

We remark also that even in the two-person case, there are games of perfect information that have no $m$-acceptable points in pure strategies. See $\S 2$ of this paper, which applies unchanged in its entirety to the non-cooperative case.

9. Equivalence of $M$-acceptability and $C$-acceptability in games of perfect information.

THEOREM 9.1. In a game $G$ of perfect information,

$$
H(M)=H(C)
$$

Proof. $H(M) \subset H(C)$ follows at once from $M \subset C$. It remains to prove

$$
H(C) \subset H(M) .
$$

Instead of proving this, we will prove a more general version that we will need later. What we need for 9.1 follows from 9.2 if we set $B=N$.

Lemma 9.2. Let $G$ be a game of perfect information. Then with each $c^{B} \in C^{B}$, we may associate an $m^{B} \in M^{B}$, such that for all $c^{N-B} \in C^{N-B}$ we have

$$
H\left(c^{B}, c^{N-B}\right)=H\left(m^{B}, c^{N-B}\right) .
$$

Proof. Fix $c^{B}$. Because of the linearity of $H$, it is sufficient to prove that there is an $m^{B}$ such that for all $p^{N-B} \in P^{N-B}$ we have

$$
H\left(c^{B}, p^{N-B}\right)=H\left(m^{B}, p^{N-B}\right) \text {. }
$$


Let $b$ be the cardinality of $B$. With each $i \in B$, we may associate an $n-b+1$ person game $G_{i}$ as follows: The players are 0 and the members of $N-B$. (Intuitively, 0 represents the coalition of all the members of $B$.) The set of pure strategies of 0 is $P^{B}$, while the set of pure strategies for a member $j$ of $N-B$ is $P^{j}$. The payoff to 0 is given by $E^{i}$, to members $j$ of $N-B$ by $E^{j}$. To avoid confusion, we will denote the payoff in $G_{i}$ by $E_{i}$, the expected payoff by $H_{i} . \quad E_{i}$ and $H_{i}$ are $((0) \cup N-B)$-vectors.

From the definition of $G_{i}$, we see that for all $p^{N-B} \in P^{N-B}$, we have

$$
\left\{\begin{array}{l}
H^{N-B}\left(c^{B}, p^{N-B}\right)=H_{i}^{N-B}\left(c^{B}, p^{N-B}\right) \\
H^{i}\left(c^{B}, p^{N-B}\right)=H_{i}^{0}\left(c^{B}, p^{N-B}\right) .
\end{array}\right.
$$

In $G_{i}, c^{B}$ is a mixed strategy of player 0 . Let $\beta^{*}$ be its behavior (see [9], §5, which will be called $\left(^{*}\right.$ ) in the sequel; Definition 16). Since $G_{i}$ depends on $i$ only because of its payoff, and since the behavior of a mixed strategy has nothing to do with the payoff, $\beta^{*}$ is independent of $i$. Since $G$ is of perfect information, so is $G_{i}$, and hence in particular, $G_{i}$ is of perfect recall. Noting that every pure strategy is also a behavior strategy, and in fact its own behavior, and applying Theorem 4 of $\left(^{*}\right)$, we obtain that for all $p^{N-B} \in P^{N-B}$,

$$
H_{i}\left(c^{B}, p^{N-B}\right)=H_{i}\left(\beta^{*}, p^{N-B}\right) .
$$

Returning to the game $G$, define behavior strategies $\beta^{i}$ for each $i \in B$ by

$$
\beta^{i}=\beta^{*} \mid \mathscr{U}^{i},
$$

where $\mathscr{U}^{i}$ is the set of information sets for player $i$.

Then from Definitions 14 and 15 of $\left(^{*}\right)$ it follows that for all $d^{N-B} \in P^{N-B}$,

$$
\left\{\begin{array}{l}
H_{i}^{0}\left(\beta^{*}, p^{N-B}\right)=H^{i}\left(\beta^{B}, p^{N-B}\right) \\
H_{i}^{N-B}\left(\beta^{*}, p^{N-B}\right)=H^{N-B}\left(\beta^{B}, p^{N-B}\right) .
\end{array}\right.
$$

Combining (2), (3), and (4), we obtain that for all $p^{N-B} \in P^{N-B}$,

$$
H^{N-B}\left(c^{N-B}, p^{N-B}\right)=H^{N-B}\left(\beta^{B}, p^{N-B}\right)
$$

and for all $i \in B, H^{i}\left(c^{N-B}, p^{N-B}\right)=H^{i}\left(\beta^{B}, p^{N-B}\right)$; that is,

$$
H\left(c^{N-B}, p^{N-B}\right)=H\left(\beta^{B}, p^{N-B}\right) .
$$

If $m^{i}$ is the mixed strategy corresponding to $\beta^{i}$ in accordance with Lemma 3 of $(*)$, then it follows from Lemma 3 and Theorem 4 of $\left(^{*}\right)$ that for all $p^{N-B} \in P^{N-B}$,

$$
H\left(\beta^{B}, p^{N-B}\right)=H\left(m^{B}, p^{N-B}\right) .
$$

Combining (5) and (6), we obtain (1). 
The following theorem will not be used in the sequel. It is included for the sake of completeness.

Corollary 9.3. In a game $G$ of perfect information

$$
E(M)=E(C) \text {. }
$$

Proof. It is clear that $E(M) \subset E(C)$. To prove $E(C) \subset E(M)$, let $c \in C$. If $\mu$ is the payoff function on $W$, we have

$$
E(P)=\mu(W)
$$

and indeed

$$
E=\mu \circ \lambda
$$

Hence if

$$
c=\sum_{p \in P}^{*} c(p) p \in C,
$$

then

$$
\begin{aligned}
H(c)= & \sum_{p \in P} c(p) \mu(\lambda(p)) \\
& \in H(C) \subset H(M) \quad(\text { by } 9.1) .
\end{aligned}
$$

It follows that there is a mixed strategy vector $m$ such that

$$
\sum_{p \in P} c(p) \mu(\lambda(p))=\sum_{p \in P}\left(\prod_{i \in N} m^{i}\left(p^{i}\right)\right) \mu(\lambda(p)) .
$$

Let us fix the coefficients $c(p)$, and consider a game $G^{\prime}$ which is the same as $G$ except for its payoff, which is such that the $\mu(w)$ form a set that is linearly independent over the field generated by the coefficients $c(p)$ over the rationals. For this game $G^{\prime}$, a mixed strategy vector $m$ may be formed that satisfies (2). Both sides of (2) can then be considered as linear combinations of distinct terms of the form $\mu(w)$, and it follows from the way we have chosen $G^{\prime}$ that the coefficients of the same terms on both sides of (2) must be equal, i.e.,

$$
\sum_{p \in \lambda^{-1}(w)} c(p)=\sum_{p \in \lambda^{-1}(w)}\left(\prod_{i \in N} m^{i}\left(p^{i}\right)\right), w \in W .
$$

Now (3) is seen to hold independent of the payoff; hence no matter how $\mu$ is defined, we may write

$$
\sum_{w \in W}^{*}\left(\sum_{p \in \lambda^{-1}(w)} c(p)\right) \mu(w)=\sum_{w \in W}^{*}\left(\sum_{p \in \lambda^{-1}(w)}\left(\prod_{\imath \in N} m^{i}\left(p^{i}\right)\right)\right) \mu(w) .
$$

(note that the outer sum is to be considered a probability distribution rather than an ordinary sum). From (4) we deduce 


$$
\sum_{p \in P}^{*} c(p) \mu(\lambda(p))=\sum_{p \in P}^{*}\left(\prod_{i \in N} m^{i}\left(p^{i}\right)\right) \mu(\lambda(p)),
$$

whence, applying (1), we obtain

$$
E(c)=\sum_{p \in P}^{*}\left(\prod_{i \in N} m^{i}\left(p^{i}\right)\right) E(p)=E(m) .
$$

This completes the proof.

COROLlaRY 9.4. In a game $G$ of perfect information,

$$
H\left(A_{m}\right) \subset H\left(A_{c}\right) \text {. }
$$

Proof. Suppose $h \notin H\left(A_{c}\right)$. Then there is a $B \subset N$, such that for all $c^{N-B} \in c^{N-B}$, there is a $c^{B} \in C^{B}$ such that

$$
H^{B}\left(c^{B}, c^{N-B}\right)>h^{B} .
$$

In particular, for all $m^{N-B} \in M^{N-B}$, there is a $c^{B} \in C^{B}$ such that

$$
H^{B}\left(c^{B}, m^{N-B}\right)>h^{B} .
$$

If we let $m^{B}$ be the mixed strategy $B$-vector associated with $c^{B}$ in accordance with Lemma 9.2, then we have

$$
H\left(m^{B}, m^{N-B}\right)=H\left(c^{B}, m^{N-B}\right) .
$$

Combining (2) and (3), we obtain that for each $m^{N-B}$, there is an $m^{B} \in M^{B}$ for which

$$
H^{B}\left(m^{B}, m^{N-B}\right)>h^{B} .
$$

Hence $h \notin H\left(A_{m}\right)$, and the corollary follows.

COROLlaRy 9.5. In a game $G$ of perfect information,

$$
H\left(A_{c}\right) \subset H\left(A_{m}\right) .
$$

Proof. Suppose $h \in H\left(A_{c}\right)$. Then for all $B \subset N$, there is a $c^{N-B} \in C^{N-B}$, such that for all $c^{B} \in C^{B}$, there is an $i \in B$ for which

$$
H^{i}\left(c^{B}, c^{N-B}\right) \leq h^{i} .
$$

Let $m^{N-B}$ be the mixed strategy $(N-B)$-vector associated with $c^{N-B}$ in accordance with Lemma 9.2. It then follows from 9.2 that for all $c^{B} \in C^{B}$,

$$
H\left(c^{B}, m^{N-B}\right)=H\left(c^{B}, c^{N-B}\right),
$$

and combining (1) and (2), we obtain that for all $c^{B} \in C^{B}$, there is an $i \in B$ for which 


$$
H^{i}\left(c^{B}, m^{N-B}\right) \leq h^{i} .
$$

In particular, for all $m^{B} \in M^{B}$, there is an $i \in B$ for which

$$
H^{i}\left(m^{B}, m^{N-B}\right) \leq h^{i},
$$

and since this holds for all $B \subset N$, it follows that $h \in H\left(A_{m}\right)$, q.e.d.

COROLlaRY 9.6. In a game $G$ of perfect information,

$$
H\left(A_{c}\right)=H\left(A_{m}\right) \text {. }
$$

COROLLARY 9.7. In a game $G$ of perfect information,

$$
A_{m}=A_{c} \cap M \text {. }
$$

Proof. If $m \in A_{m}$, then certainly

$$
m \in M \text {. }
$$

But from 9.5 it follows that $H(m) \in H\left(A_{c}\right)$. Since among $c$-strategy vectors, the property of $c$-acceptability is a global one, depending only on the payoff, it follows that

$$
m \in A_{c} \text {. }
$$

Combining (1) and (2), we obtain $m \in A_{c} \cap M$.

Next, let $c \in A_{c} \cap M$. Then $c \in M$. We also have $H(c) \in H\left(A_{m}\right)$, and since among $m$-strategy vectors, the property of $m$-acceptability is a global one, depending only on the payoff, it follows that

$$
c \in A_{m} \text {. }
$$

This completes the proof.

Because of 9.6 and 9.7, we are justified in dropping the qualifying prefix from the word "acceptable" when discussing games of perfect information.

10. Supergame strategies in the non-cooperative case. A supergame strategy vector for a non-cooperative game is the same as a supergame strategy vector for a cooperative game, except that coalitions are forbidden. Formally, we have

DEFINITION 10.1. A supergame $m$-strategy $f^{i}$ for player $i$ is a supergame c-strategy for which

$$
e\left(f_{k}^{i}(y)\right)=(i)
$$

for all $k \geq 0$ and $y \in J_{1}^{i} \times \cdots \times J_{k}^{i}$. 
The following theorem follows at once from 10.1:

Theorem 10.2. For a supergame m-strategy vector $f$, we have

$$
c\left(f_{k}(y)\right) \in M
$$

for all $k \geq 0$ and $y \in J_{1} \times \cdots \times J_{k}$.

Parallel to the definition of strong equilibrium $c$-point for cooperative games ( $\$ 7$ of $[1]$ ), we may make the following definition for non-cooperative games:

DEFINITION 10.3. Let $f$ be a summable supergame $m$-strategy vector. $f$ is a strong equilibrium m-point if there is no $B \subset N$ for which there is a supergame m-strategy vector $g$ satisfying 7.1 and 7.2 of [1].

The set of strong equilibrium $m$-points will be denoted by $S_{m}$. As in [1], it is possible to replace 7.2 of [1] by 7.3 of [1]. The set of points thus obtained will be denoted by $\widetilde{S}_{m}$.

LEMMA 10.4. $F_{p} \cap S_{c} \subset S_{m}$.

Proof. Let

$$
f \in F_{p} \cap S_{c} .
$$

Since $f \in F_{p}$, it follows in particular that $f$ is a summable supergame $m$-strategy vector. Suppose

$$
f \notin S_{m} .
$$

Then there is a $B \subset N$ and a supergame $m$-strategy vector $g$ satisfying 7.1 and 7.2 of $\lfloor\mathbf{1}\rfloor$. Since every supergame $m$-strategy vector is also a supergame $c$-strategy vector, it follows that there is a supergame $c$-strategy vector $g$ satisfying 7.1 and 7.2 of $[\mathbf{1}]$. Hence

$$
f \notin S_{c},
$$

contradicting (1). Hence (1) implies the falsity of (2), and our result is proved.

Lemma $10.5 . \quad F_{p} \cap S_{m} \subset S_{p}$.

Proof. The proof is word for word the same as that of the second part of Theorem 3.11 (the part beginning with the word "conversely"; the proof is given before the proof of the first part), except that the two occurrences of the prefix "c-" must be replaced by prefixes " $m$-". It is also necessary to remember that since $g$ is pure, it is in particular mixed. 
THEOREM 10.6. $\quad F_{p} \cap S_{m}=S_{p}$.

Proof. We have

$$
\begin{aligned}
S_{p}= & F_{p} \cap S_{c} \quad(\text { by } 3.11) \\
& \subset S_{m} \quad(\text { by 10.4) }
\end{aligned}
$$

Since

$$
S_{p} \subset F_{p},
$$

it follows that

$$
S_{p} \subset F_{p} \cap S_{m}
$$

Combining this with 10.5 , we obtain 10.6 .

THEOREM 10.7. $\quad F_{p} \cap \widetilde{S}_{m}=\widetilde{S}_{p}$.

Proof. The proof is similar to that of 10.6 .

11. The main theorem for non-cooperative games.

THEOREM 11.1. In a game $G$ of perfect information,

$$
H\left(A_{m}\right)=H\left(S_{p}\right)=H\left(\widetilde{S}_{p}\right) .
$$

In particular, $h$ is an m-acceptable payoff vector in $G$, if and only if there is a strong equilibrium m-point $f$ in supergame pure strategies for which

$$
H(f)=h
$$

Proof. The first part follows from 5.3 and 9.6. The second part follows from 10.6 and from the first part.

COROLLARY 11.2. Every stable game of perfect information has strong equilibrium m-points in supergame pure strategies.

Finally, we remark that the discussion of $\S 6$ applies unchanged to the non-cooperative case.

\section{REFERENCES}

1. R. J. Aumann, Acceptable points in general cooperative n-person games, Contributions to the Theory of Games IV, Annals of Mathematics Study 40, Princeton University Press, 1959, pp. 287-324.

2. C. Berge, Théorie générale des Jeux a n personnes, Mémorial des Sciences Mathématiques 138, 1957.

3. Topological games with perfect information, Contributions to the Theory of 
Games III, Annals of Mathematics Study 39, Princeton University Press, 1957, pp. 165-178. 4. N. Dalkey, Equivalence of information patterns and essentially determinate games, Contributions to the Theory of Games II, Annals of Mathematics Study 28, Princeton University Press, 1953, pp. 217-244.

5. H. Everett, Recursive games, Contributions to the Theory of Games III, Annals of Mathematics Study 39, Princeton University Press, 1957, pp. 47-78.

6. D. Gale and F. M. Stewart, In finite games with perfect information, Contributions to the Theory of Games II, Annals of Mathematics Study 28, Princeton University Press, 1953, pp. 245-266.

7. D. Gillette, Stochastic games with zero stop probabilities, Contributions to the Theory of Games III, Annals of Mathematics Study 39, Princeton University Press, 1957, pp. 179187.

8. D. B. Gillies, Some theorems on n-person games, Ph. D. Thesis, Princeton University, 1953.

9. H. W. Kuhn, Extensive games and the problem of information, Contributions to the Theory of Games II, Annals of Mathematics Study 28, Princeton University Press, 1953, pp. 193-216.

10. Lectures on the theory of games, issued as a report of the Logistics Research Project, Princeton University, 1952.

11. O. Morgenstern, and J. von Neumann, Theory of games and economic behavior, Princeton University Press, 1953.

12. J. F. Nash, Non-cooperative games, Ann. Math. 54 (1951), 286-295.

13. L. S. Shapley, Stochastic games, Proc. Nat. Acad. Sci. 39 (1953), 1095-1100.

14. P. Wolfe, The strict determinateness of certain infinite games, Pacific J. Math. 5 (1955), 891-897.

The Hebrew University, Jerusalem, Israel 



\section{PACIFIC JOURNAL OF MATHEMATICS}

\section{EDITORS}

David Gilbarg

Stanford University

Stanford, California

F. H. Brownell

University of Washington

Seattle 5, Washington
A. L. Whiteman

University of Southern Californıa Los Angeles 7, California

L. J. PaIge

University of California

Los Angeles 24, California

\section{ASSOCIATE EDITORS}
E. F. BECKENBACH
T. M. CHERRY
D. DERRY

E. HEWITT
A. HORN
L. NACHBIN
M. OHTSUKA

H. L. ROYDEN

M. M. SCHIFFER
E. SPANIER

E. G. STRAUS

F. WOLF

\section{SUPPORTING INSTITUTIONS}

\author{
UNIVERSITY OF BRITISH COLUMBIA \\ CALIFORNIA INSTITUTE OF TECHNOLOGY \\ UNIVERSITY OF CALIFORNIA \\ MONTANA STATE UNIVERSITY \\ UNIVERSITY OF NEVADA \\ NEW MEXICO STATE UNIVERSITY \\ OREGON STATE COLLEGE \\ UNIVERSITY OF OREGON \\ OSAKA UNIVERSITY \\ UNIVERSITY OF SOUTHERN CALIFORNIA
}

\author{
STANFORD UNIVERSITY \\ UNIVERSITY OF TOKYO \\ UNIVERSITY OF UTAH \\ WASHINGTON STATE COLLEGE \\ UNIVERSITY OF WASHINGTON \\ AMERICAN MATHEMATICAL SOCIETY \\ CALIFORNIA RESEARCH CORPORATION \\ HUGHES AIRCRAFT COMPANY \\ SPACE TECHNOLOGY LABORATORIES \\ NAVAL ORDNANCE TEST STATION
}

Mathematical papers intended for publication in the Pacific Journal of Mathematics should be typewritten (double spaced), and the author should keep a complete copy. Manuscripts may be sent to any one of the four editors. All other communications to the editors should be addressed to the managing editor, L. J. Paige at the University of California, Los Angeles 24, California.

50 reprints per author of each article are furnished free of charge; additional copies may be obtained at cost in multiples of 50 .

The Pacific Journal of Mathematics is published quarterly, in March, June, September, and December. The price per volume (4 numbers) is $\$ 12.00$; single issues, $\$ 3.50$. Back numbers are available. Special price to individual faculty members of supporting institutions and to individual members of the American Mathematical Society: $\$ 4.00$ per volume; single issues, $\$ 1.25$.

Subscriptions, orders for back numbers, and changes of address should be sent to Pacific Journal of Mathematics, 2120 Oxford Street, Berkeley 4, California.

Printed at Kokusai Bunken Insatsusha (International Academic Printing Co., Ltd.), No. 6, 2-chome, Fujimi-cho, Chiyoda-ku, Tokyo, Japan.

PUBLISHED BY PACIFIC JOURNAL OF MATHEMATICS, A NON-PROFIT CORPORATION

The Supporting Institutions listed above contribute to the cost of publication of this Journal, but they are not owners or publishers and have no responsibility for its content or policies. 


\section{Pacific Journal of Mathematics}

\section{Vol. 10, No. $2 \quad$ October, 1960}

Maynard G. Arsove, The Paley-Wiener theorem in metric linear spaces ........

Robert (Yisrael) John Aumann, Acceptable points in games of perfect

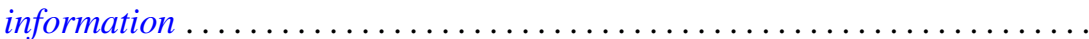

A. V. Balakrishnan, Fractional powers of closed operators and the semigroups generated by them ... . . . . . . . . . . . . . . . . . . . . . . . . . . . . 419

Dallas O. Banks, Bounds for the eigenvalues of some vibrating systems . . . . . 439

Billy Joe Boyer, On the summability of derived Fourier series . . . . . . . . . . . 475

Robert Breusch, An elementary proof of the prime number theorem with

remainder term ...................................

Edward David Callender, Jr., Hölder continuity of $n$-dimensional

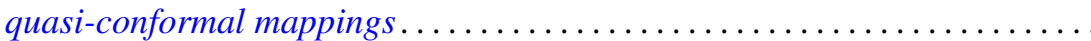

L. Carlitz, Note on Alder's polynomials ......................... 517

P. H. Doyle, III, Unions of cell pairs in $E^{3} \ldots \ldots \ldots \ldots \ldots \ldots \ldots \ldots \ldots \ldots \ldots \ldots . \ldots 21$

James Eells, Jr., A class of smooth bundles over a manifold . . . . . . . . . . . . 525

Shaul Foguel, Computations of the multiplicity function . . . . . . . . . . . . . . 539

James G. Glimm and Richard Vincent Kadison, Unitary operators in

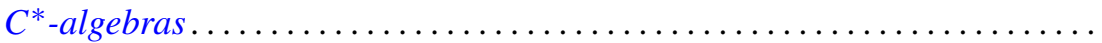

Hugh Gordon, Measure defined by abstract $L_{p}$ spaces . . . . . . . . . . . 557

Robert Clarke James, Separable conjugate spaces ....................

William Elliott Jenner, On non-associative algebras associated with bilinear forms

Harold H. Johnson, Terminating prolongation procedures

John W. Milnor and Edwin Spanier, Two remarks on fiber homotopy type .

Donald Alan Norton, A note on associativity . .

Ronald John Nunke, On the extensions of a torsion module.

Joseph J. Rotman, Mixed modules over valuations rings . . . . .

A. Sade, Théorie des systèmes demosiens de groupoï des . .

Wolfgang M. Schmidt, On normal numbers . .

661

Berthold Schweizer, Abe Sklar and Edward Oakley Thorp, The metrization of

statistical metric spaces

John P. Shanahan, On uniqueness questions for hyperbolic differential

equations

A. H. Stone, Sequences of coverings

Edward Oakley Thorp, Projections onto the subspace of compact operators

L. Bruce Treybig, Concerning certain locally peripherally separable spaces

Milo Wesley Weaver, On the commutativity of a correspondence and a

permutation

David Van Vranken Wend, On the zeros of solutions of some linear complex

differential equations. 